\title{
Hyper-spectral characteristics of rolled-leaf desert vegetation in the Hexi Corridor, China
}

\author{
WEI Huaidong ${ }^{1,2}$, YANG Xuemei ${ }^{2,3^{*}}$, ZHANG Bo ${ }^{1}$, DING Feng ${ }^{2}$, ZHANG Weixing ${ }^{2}$, LIU \\ Shizeng ${ }^{2}$, CHEN Fang ${ }^{2}$ \\ ${ }^{1}$ College of Geography and Environment Science, Northwest Normal University, Lanzhou 730070, China; \\ ${ }^{2}$ State Key Laboratory Breeding Base of Desertification and Aeolian Sand Disaster Combating, Gansu Desert Control Research \\ Institute, Lanzhou 730070, China; \\ ${ }^{3}$ Key Laboratory of Remote Sensing of Gansu Province, Northwest Institute of Eco-Environment and Resources, Chinese \\ Academy of Sciences, Lanzhou 730000, China
}

\begin{abstract}
Desert plants survive harsh environment using a variety of drought-resistant structural modifications and physio-ecological systems. Rolled-leaf plants roll up their leaves during periods of drought, making it difficult to distinguish between the external structures of various types of plants, it is therefore necessary to carry out spectral characteristics analysis for species identification of these rolled-leaf plants. Based on hyper-spectral data measured in the field, we analyzed the spectral characteristics of seven types of typical temperate zone rolled-leaf desert plants in the Hexi Corridor, China using a variety of mathematical transformation methods. The results show that: (1) during the vigorous growth period in July and August, the locations of the red valleys, green peaks, and three-edge parameters, namely, the red edge, the blue edge, and the yellow edge of well-developed rolled-leaf desert plants are essentially consistent with those of the majority of terrestrial vegetation types; (2) the absorption regions of liquid water, i.e., 1400-1500 and 1600-1700 nm, are the optimal bands for distinguishing various types of rolled-leaf desert plants; (3) in the leaf reflectance regions of 700-1250 $\mathrm{nm}$, which is controlled by cellular structure, it is difficult to select the characteristic bands for differentiation rolled-leaf desert vegetation; and (4) after processing the spectral reflectance curves using a first-order differential, the envelope removal method, and the normalized differential ratio, we identify the other characteristic bands and parameters that can be used for identifying various types of temperate zone rolled-leaf desert plants, i.e., the 510-560, 650-700 and 1330-1380 nm regions, and the red edge amplitude. In general, the mathematical transformation methods in the study are effective tools to capture useful spectral information for species identification of rolled-leaf plants in the Hexi Corridor.
\end{abstract}

Keywords: rolled-leaf desert vegetation; spectral characteristics; mathematical transformation; vegetation identification; Hexi Corridor

Citation: WEI Huaidong, YANG Xuemei, ZHANG Bo, DING Feng, ZHANG Weixing, LIU Shizeng, CHEN Fang. 2019. Hyper-spectral characteristics of rolled-leaf desert vegetation in the Hexi Corridor, China. Journal of Arid Land, 11(3): 332-344. https://doi.org/10.1007/s40333-019-0013-x

\section{Introduction}

The study of spectral reflectance and spectral determination of different vegetation types using remote sensing forms the practical basis for understanding the physical and chemical properties of

\footnotetext{
*Corresponding author: YANG Xuemei (E-mail: yxm9693@163.com)

Received 2017-11-02; revised 2018-06-13; accepted 2018-06-20

(C) Xinjiang Institute of Ecology and Geography, Chinese Academy of Sciences, Science Press and Springer-Verlag GmbH Germany, part of Springer Nature 2019
} 
vegetation, for example, vegetation classification, species identification, monitoring the ecological environment and vegetation (Fang et al., 2017). To date, the use of hyper-spectral technology has allowed for significant achievements in vegetation identification, phenological monitoring, and biomass and vegetation coverage inversion (Gates et al., 1965; Li, 2008; Gai et al., 2011; Yang et al., 2016; Liu et al., 2017; Meyer et al., 2017). The specialized study of spectral determination and characteristics of desert vegetation can provide the basis for species identification, remote sensing classification, and large-scale ecosystem monitoring. For example, Zhao et al. (2009) and Zhang et al. (2012) proposed the optimal hyper-spectral bands for identification of vegetation in the oasis salinization-desert zone in the middle reaches of the Tarim River. Other researchers carried out the studies on the spectral characteristics of the main vegetation species in the Seriphidium transiliense desert grassland in Xinjiang, including grassland degradation areas and areas under enclosure before and after its installation, and identified the main vegetation species in combination with vegetation indices (He et al., 2014; Jin et al., 2014; Xia et al., 2015; Wei et al., 2016). Zhang et al. (2006) determined the spectral reflectance of the canopy and leaves in saline lowland meadow, extremely in arid desert meadow, and desert shrub environments of the desert grassland in Anxi County, located in the arid inland region of Northwest China, and analyzed the main factors influencing the various spectral characteristics of desert-meadow vegetation species. Based on hyper-spectral image analysis of the Shiyang River Basin, located in the Hexi Corridor, Zhang et al. (2012) proposed that since the red edge, green peak, and absorption valley locations of blue and red light remain consistent for different vegetation coverage conditions, and the spectrum absorption characteristics of visible light bands change systematically with increasing coverage, which can be serve as a basis for extracting or determining vegetation coverage. Qian et al. (2013) analyzed typical desert vegetation in Xinjiang and suggested that because of the low vegetation coverage rate, the red edge characteristics, which are affected by the underlying surface, decreased with the decline of vegetation coverage inversion. In addition, several studies explored desert vegetation structure, water content, chlorophyll content, canopy transpiration, and other sensitive band regions that have practical significance in desert ecosystem monitoring (Zhao, 2011; Jin et al., 2016; Liu et al., 2017).

There are many methods to identify plant species using spectral discrimination, for example, Schmidt et al. (2003) discriminated 27 saltmarsh vegetation species in coastal wetlands based on the Bhattacharyya $(\mathrm{BH})$ and the Jeffries-Matusita (JM) distance measures. The random forest algorithm and a forward variable selection technique were used to identify optimal wavelengths for discriminating the grass species (Mansour et al., 2012). Three-tier hierarchical techniques of one-way analysis of variance (ANOVA), stepwise discriminant function analysis, and canonical function analysis were also used for discriminating increaser species in degraded rangeland (Mansour et al., 2013). ANOVA, classification and regression trees, and JM distance method were used to spectrally discriminate papyrus from other species as well as to reduce and subsequently select optimal bands for the potential discrimination of papyrus (Adam et al., 2009). Comparatively speaking, the common mathematical transformation methods of first-order differential, continuum removal normalization and normalized differential ratio, and three-edge parameters are more widely used in spectral analysis methods for the identification of plant species (Cao et al., 2013; An et al., 2014; Yang et al., 2014; Zarco-Tejada et al., 2018).

Desert plants adopt structural changes in their branches and leaves in order to reduce transpiration in arid desert environments. Some plants have become leafless through foliage reduction or degradation, while others possess succulent stems or leaves that allow for water storage. Others yet have developed protective tissue on their stems (Zhou et al., 2013). Some plants with leaves that can roll up to resist drought, meanwhile, it is hard to distinguish them because of their similarities in structure, such as the rolled-leaf Poa annua and Elymus dahuricus species. Therefore, it is necessary to study the spectral characteristics of various types of desert plants. However, very few studies have focused on a particular type of desert plants. Furthermore, some studies on desert plants focus mainly on spectral observation and analysis; very few have been carried out for species identification, especially for rolled-leaf plants. In this study, we 
selected seven types of typical rolled-leaf desert plants, i.e., Poa annua, Stipa purpurea, Stipa grandis, Elymus dahuricus, Achnatherum splendens, Stipa glareosa, and Agropyron cristatum in the Hexi Corridor, China and analyzed their spectral reflectance characteristics for identifying these plant species. The results can be used as a data base for the identification and classification of temperature desert vegetation.

\section{Study area and methods}

\subsection{Study area}

The Hexi Corridor $\left(37^{\circ} 17^{\prime}-42^{\circ} 48^{\prime} \mathrm{N}, 93^{\circ} 23^{\prime}-104^{\circ} 12^{\prime} \mathrm{E}\right)$ is located in the central region of the Eurasian continent and the eastern part of the desert region of Northwest China., which is mainly located in the western and northern parts of Kumtag, Badain Jaran and Tengger deserts, Northwest China. The region is mainly composed of alluvial and fluvial plains, shallow mountains, oases, deserts, and Gobi. The average annual precipitation in the region is approximately $140 \mathrm{~mm}$, gradually decreasing from southeast to northwest and falling below 50 $\mathrm{mm}$ in some areas. The potential evaporation exceeds $1000 \mathrm{~mm}$. Because of the diversity and typicality of its vegetation, the Hexi Corridor is a natural laboratory for studies on the spectral characteristics of temperate vegetation (Zhou, 2013). The vegetation in this region is categorized according to the main strategies that the plants use to cope with the arid environment of the temperate zone. The main categories are: (1) plants with relatively low water content and extremely drought-resistant thin leaves, (2) succulent plants with fleshy leaves and stems, and plants with succulent stems and extremely atrophied leaves, and (3) plants with leaves that can roll up to resist drought.

\subsection{Spectral data in field}

In this study, the spectral reflectance of each plot was measured under clear skies between 10:00 (GMT) and 14:00 with an ASD spectrometer (Fieldspec ${ }^{\circledR} 4$ Standard-Res, USA), which covered the wavelengths of $350-2500 \mathrm{~nm}$ with a $1.5-\mathrm{nm}$ sampling interval. The field spectral data were collected between 25 July and 8 August, 2015, when the weather was sunny and cloudless with good light and low wind, and the measurements were obtained during the day between 10:00 and 14:00. We performed a standard whiteboard calibration before recording the measurements to minimize the influence of soil back ground on spectral measurement and decrease the scan diameter to lower than $10 \mathrm{~cm}$ based on the field view of the spectrometer of $25^{\circ}$. Moreover, the vertical downward distance between the sensor and vegetation canopy was approximately 10-20 cm. Fifty well-developed specimens from each rolled-leaf desert plant type were selected for spectral measurement in the whole study area. To ensure the quality of spectral information collected in the field, we measured five spectral bands for each plant, and took the averaged values as the reflectance spectra. Of the obtained samples, 30 plants were used for analyzing the spectral characteristics of rolled-leaf desert vegetation, and 20 plants were used as test samples for identification. After recording the spectra, the obtained data were compared with the standard spectral reflectance curve and, after any invalid original data were removed, quadrat sampling of the plants was carried out accordingly.

\subsection{Spectral characteristic parameter extraction}

\subsubsection{Data preprocessing}

Surface spectrometers produce a significant amount of noise, which can influence the post-processing analysis. Therefore, we used a Savitzky-Golay filter, which is based on least squares polynomial fitting across a moving window, to smooth the data and remove the noise. Considering the collected data, the spectral reflectance of the 400-1800 nm bands is normal, whereas the 1800-2500 nm bands show obvious noise, which masks the spectral reflectance signal of the plants, and is thus eliminated.

The visible light spectral reflectance depends mainly on the plant chlorophyll content and the vegetation coverage. The green peak refers to the maximum spectral reflectance parameter in 
the range of 510-560 $\mathrm{nm}$, which is mainly due to the strong absorption of blue and yellow light by the plant pigment, thus showing up as a strong reflection peak in the green band. The red valley refers to the lowest spectral reflectance in the range of 650-690 nm (Table 1).

\subsubsection{Three-edge parameters}

The three-edge parameters utilize spectral differential techniques, including mathematical simulation and calculation of different orders of differential values of the reflectance spectrum, to quickly determine the wavelength position of the spectrum bending point and the maximum and minimum reflectance. The first derivative reflectance (first-order differential reflectance, FDR) can help to eliminate the atmospheric and soil background effects from the spectral data, by using the following equation (An, 2014):

$$
\operatorname{FDR}_{\lambda(j)}=\left(R_{\lambda(j+1)}-R_{\lambda(j)}\right) / \Delta \lambda,
$$

where $\operatorname{FDR}_{\lambda(j)}$ is the first-order differential reflectance at a wavelength midpoint between bands $j$ and $j+1 ; R_{\lambda(j+1)}$ is the reflectance at band $j+1 ; R_{\lambda(j)}$ is the original reflectance at band $j$; and $\Delta \lambda$ is the difference in wavelength between $j$ and $j+1$.

The three-edge parameters comprise the red edge, yellow edge, and blue edge (Table 1), which are important spectral parameters used to distinguish the various species of plants. Chlorophyll content is the main factor influencing the red edge, which is an important band indicator describing the pigmentation status and the health condition of vegetation.

Table 1 Spectral characteristic parameters

\begin{tabular}{ll}
\hline \multicolumn{1}{c}{ Type } & \multicolumn{1}{c}{ Definition } \\
\hline Red edge amplitude & $\begin{array}{l}\text { The maximum first-order differential reflectance in the } 680-760 \mathrm{~nm} \text { regions } \\
\text { The wavelength corresponding to the maximum first-order differential reflectance in the } \\
680-760 \mathrm{~nm} \text { regions } \\
\text { Red edge position }\end{array}$ \\
$\begin{array}{l}\text { The maximum first-order differential reflectance in the } 560-640 \mathrm{~nm} \text { regions } \\
\text { The wavelength corresponding to the maximum first-order differential reflectance in the }\end{array}$ \\
$\begin{array}{l}560-640 \mathrm{~nm} \text { regions } \\
\text { Yellow edge position }\end{array}$ & $\begin{array}{l}\text { The maximum first-order differential reflectance in the } 490-530 \mathrm{~nm} \text { regions } \\
\text { The wavelength corresponding to the maximum first-order differential reflectance in the }\end{array}$ \\
Blue edge position & $490-530 \mathrm{~nm}$ regions \\
Red valley & The lowest spectral reflectance in the $650-690 \mathrm{~nm}$ regions \\
Green peak & The maximum spectral reflectance parameter in the $510-560 \mathrm{~nm}$ regions
\end{tabular}

\subsubsection{Envelope removal method}

The envelope removal method, also known as the continuum removal normalization method, can amplify the absorption or reflectance peak values and enable the comparison of the spectral absorption characteristics using a common baseline. From the reflectance spectrum curves, suitable bands are selected on the absorption valley, and the band endpoints are set as the start and end points of absorption. The continuous line between the start and end points is called the hull. The envelope removal value on each band is equal to the reflectance value of the band divided by the hull value. The use of spectral curves processed with the envelope removal method amplifies the absorption bands (Schmidt and Skidmore, 2003). The specific calculation process is as follows:

$$
\begin{gathered}
C R_{i}=R_{i} / R_{H i}, \\
R_{H i}=R_{\text {start }}+K \times\left(r_{i}-r_{\text {start }}\right), \\
K=\left(R_{\text {end }}-R_{\text {start }}\right) /\left(r_{\text {end }}-r_{\text {start }}\right),
\end{gathered}
$$

where $C R_{i}$ is the envelope value corresponding to band $i ; R_{i}$ is the spectral reflectance corresponding to band $i ; R_{H i}$ is the hull value corresponding to band $i$; $K$ is the hull slope between the endpoints of absorption; $R_{\text {start }}$ and $R_{\text {end }}$ are the reflectances at the absorption start and end 
points, respectively; and $r_{\text {start }}$ and $r_{\text {end }}$ are the wavelengths at the absorption start and end points, respectively.

\subsubsection{Normalized differential ratio}

The first-order differential can extract the slope information of the spectral curve and weaken the noise interference of the soil background effect, whereas normalization can further highlight the details of spectral characteristics. In this study, we used the first-order differential of the normalized spectrum as the basis, and divided it by the maximum value of the first-order differential, which can further enhance the spectral details and remove the soil background interference. This algorithm is called the normalized differential ratio (An et al., 2014), and its calculation process is as follows:

$$
\hat{r}=\left(r-\mu_{r}\right) / \sigma_{r},
$$

where $\hat{r}$ is the normalized spectral value; $r$ is the original spectral reflectance; and $\mu_{r}$ and $\sigma_{r}$ are the spectral average value and the standard deviation, respectively.

\section{Results}

\subsection{Reflectance spectrum}

Figure 1 shows the reflectance spectrum curves of the seven typical rolled-leaf desert plant types in the Hexi Corridor in late July. It can be seen that, although the spectral curve trends are essentially the same in the 400-1800 nm regions, subtle differences exist. In the 1020-1120 nm regions (located in the leaf reflectance regions of 700-1250 nm, which is controlled by the cellular structure), Agropyron cristatum has the highest reflectance, while Achnatherum splendens has the lowest. In the 1400-1500 $\mathrm{nm}$ regions (located in the leaf reflectance regions of 1250-2300 $\mathrm{nm}$ ), with the exception of Agropyron cristatum and Stipa grandis, there are obvious differences in the reflectance of all the other plants, with Stipa glareosa having the highest reflectance, followed in order by Stipa purpurea, Poa annua, Agropyron cristatum, Stipa grandis, and Elymus dahuricus, with Achnatherum splendens having the lowest reflectance. In general, in the leaf reflectance regions controlled by the cellular structure and water content, Achnatherum splendens has the lowest reflectance, which indicates optimal distinguishability. In addition, in the 1600-1700 $\mathrm{nm}$ regions, there are also significant differences in the reflectance of different plant types.

The green peaks and red valleys of the seven types of typical rolled-leaf desert plants in the Hexi Corridor are located in the 510-560 and 650-690 nm regions, respectively (Fig. 1). In the visible light band, the green peaks average less than $20 \%$, of which the differences between Stipa glareosa and Stipa purpurea are the smallest, and the differences between Stipa grandis and

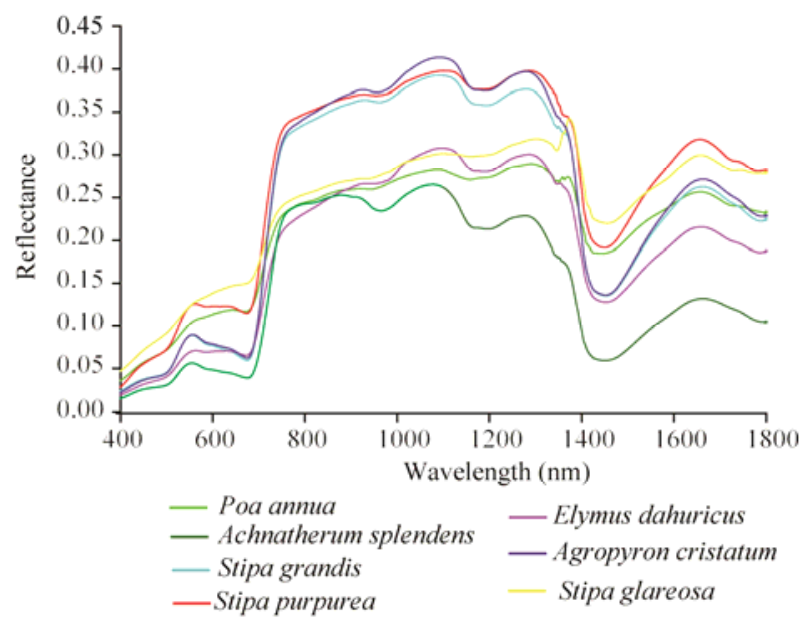

Fig. 1 Hyper-spectral curves of the typical rolled-leaf desert plant species in the Hexi Corridor 
Agropyron cristatum are also the smallest. Achnatherum splendens has the lowest green peak value, followed by Elymus dahuricus. With the exception of Achnatherum splendens and Stipa glareosa, which have the lowest and highest red valley amplitudes, respectively, the red valleys of the other plant types showed less distinctions.

\subsection{Three-edge parameters}

The first-order differential spectral curves of rolled-leaf plants show that the three-edge positions of various plants are similar (Fig. 2). In contrast to the blue edge and yellow edge amplitude regions, the distinguishability of the rolled-leaf desert plant types in the red edge amplitude region is relatively good, with the exception of Stipa grandis and Agropyron cristatum. The red edge amplitude order is as follows: Stipa purpurea $>$ Achnatherum splendens $>$ Elymus dahuricus $>$ Poa annua $>$ Stipa glareosa (Table 2). In addition, as shown in Figure 2, at the $1400( \pm 50) \mathrm{nm}$ band location, obvious spectral valleys appear and the differences between spectral characteristics are relatively clear for all plant types, among which Poa annua has the highest valley value, followed by Achnatherum splendens, Elymus dahuricus, Stipa glareosa, and Stipa purpurea. Stipa grandis and Agropyron cristatum have the lowest valley values.

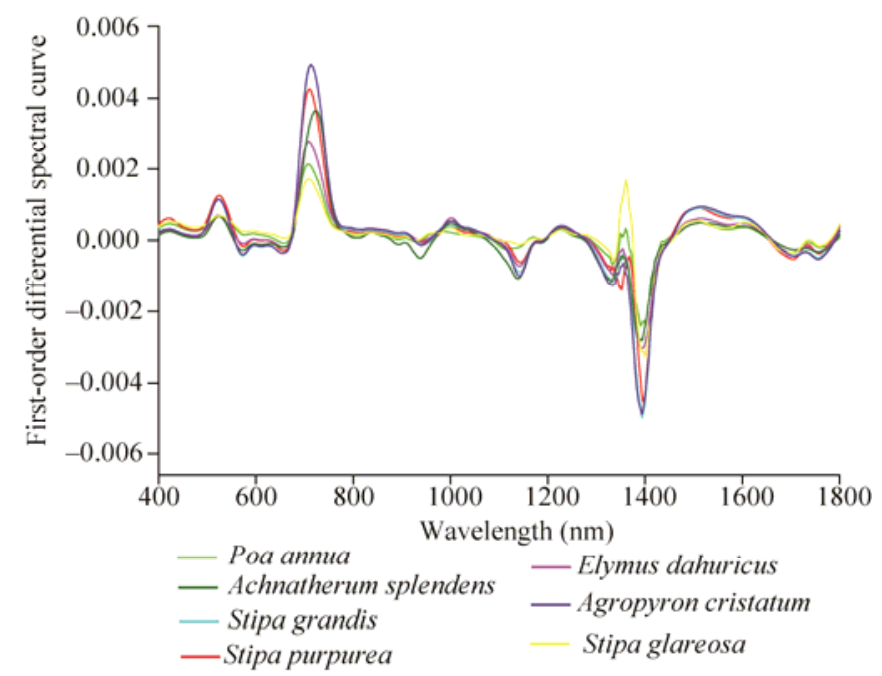

Fig. 2 First-order differential spectral curves of the typical rolled-leaf desert plant species in the Hexi Corridor

Table 2 Spectral parameters of the typical rolled-leaf desert plant species $\left(\times 10^{-3}\right)$

\begin{tabular}{lccccccc}
\hline $\begin{array}{l}\text { Spectral } \\
\text { parameter }\end{array}$ & $\begin{array}{c}\text { Agropyron } \\
\text { cristatum }\end{array}$ & $\begin{array}{c}\text { Stipa } \\
\text { grandis }\end{array}$ & $\begin{array}{c}\text { Achnatherum } \\
\text { splendens }\end{array}$ & $\begin{array}{c}\text { Elymus } \\
\text { dahuricus }\end{array}$ & Stipa glareosa & Poa annua & Stipa purpurea \\
\hline Red valley & 63.60 & 60.80 & 39.80 & 66.00 & 147.60 & 117.40 & 115.70 \\
Green peak & 90.40 & 89.70 & 56.90 & 71.30 & 127.60 & 106.30 & 126.40 \\
$\begin{array}{l}\text { Red edge } \\
\text { amplitude }\end{array}$ & 4.80 & 4.80 & 3.80 & 2.80 & 1.80 & 2.20 & 4.20 \\
$\begin{array}{l}\text { Blue edge } \\
\text { amplitude }\end{array}$ & 1.20 & 1.20 & 0.70 & 0.80 & 0.70 & 0.70 & 1.30 \\
$\begin{array}{l}\text { Yellow edge } \\
\text { amplitude }\end{array}$ & 0.40 & 0.30 & 0.20 & 0.30 & 0.50 & 0.50 & 0.60 \\
\hline
\end{tabular}

Based on the three-edge amplitudes, green peaks and red valley parameters of the seven types of typical rolled-leaf desert plants, we found that: (1) although the appearances of Stipa grandis and Agropyron cristatum are different, their spectral characteristics are similar; (2) with the exception of Stipa glareosa and Stipa purpurea at the green peak locations and Agropyron 
cristatum and Stipa grandis at the red edge locations, whose spectral characteristics are rather indistinguishable, the green peaks and the red edges are ideal spectral parameters for distinguishing among these seven types of rolled-leaf desert plants; and (3) in the regions where leaf reflectance is controlled by the water content, Achnatherum splendens has the lowest reflectance, especially in the $1400( \pm 50) \mathrm{nm}$ reflectance region of the first-order differential, and the different plant species show relatively high heterogeneity, which can help in distinguishing between Agropyron cristatum and Stipa grandis.

\subsection{Spectral absorption characteristics}

As shown in Figure 3, after the reflectance curves are processed using the envelope removal method, the spectral characteristics of the visible-light absorption valleys (510-560 and 600-700 $\mathrm{nm}$ ), the green peaks, and the 1400-1500 $\mathrm{nm}$ regions are amplified. The statistical results (Fig. 4) show that the maximum value occurs in 510-560 nm and the minimum value occurs in 650-700 $\mathrm{nm}$. In addition, the envelope removal values of the different plant species are consistently ordered as follows: Stipa glareosa $>$ Poa annua $>$ Stipa purpurea $>$ Elymus dahuricus $>$ Achnatherum splendens. Achnatherum splendens continues to show the lowest value in the 1400-1500 nm regions. Further, Stipa grandis and Agropyron cristatum are not easily distinguishable in these specific regions.

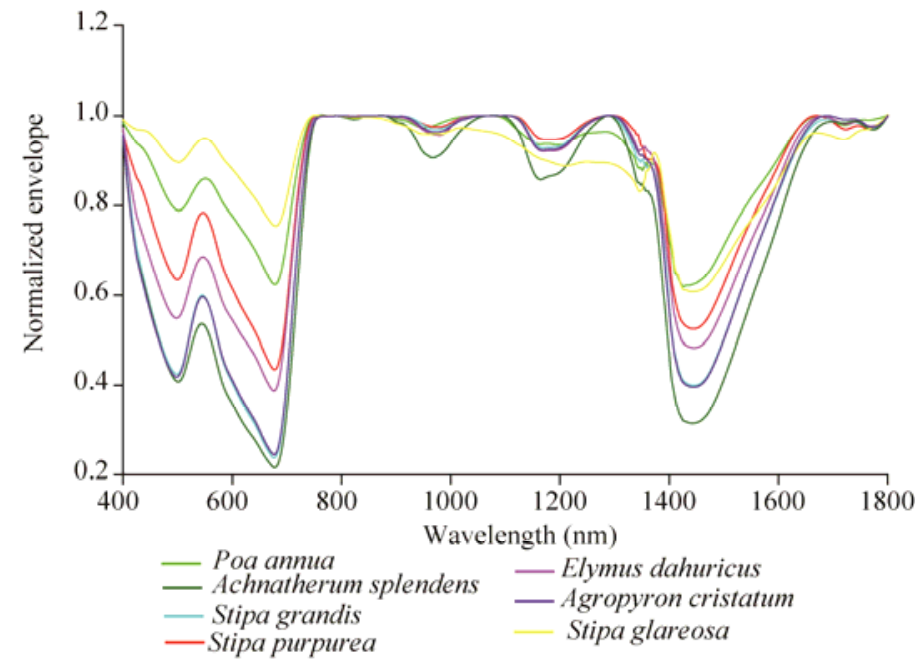

Fig. 3 Normalized envelopes of the typical rolled-leaf desert plant species in the Hexi Corridor

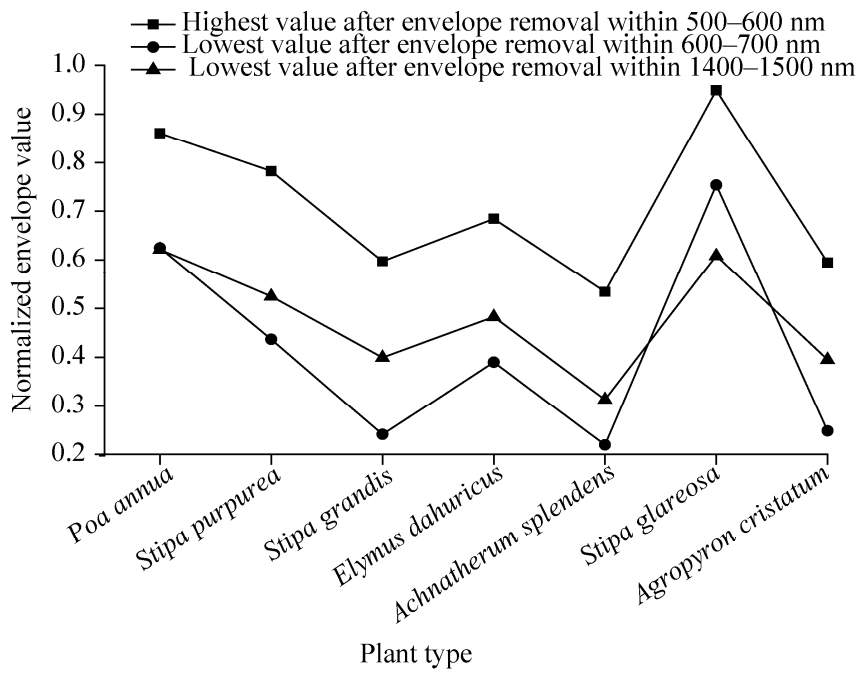

Fig. 4 Spectral absorption characteristics of the typical rolled-leaf desert plant species in the Hexi Corridor 


\subsection{Normalized differential ratio}

In order to remove the soil background effects and explore the spectral characteristics of rolled-leaf desert vegetation, we used the normalized differential ratio to process the spectral data (Fig. 5). The results show that the vegetation spectral characteristics are relatively obvious in the 1330-1380 nm regions, in which Stipa glareosa has the highest normalized differential ratio, followed by Poa annua. In the $1000( \pm 50) \mathrm{nm}$ region, Elymus dahuricus has the highest differential ratio. In general, the field measurements are carried out during the vigorous vegetation growth period and the measured plants are healthy. Moreover, the vertical downward distance between the sensor and the vegetation canopy is approximately $10-20 \mathrm{~cm}$, ensuring the maximum reduction in the soil background interference effect. Therefore, no obvious improvement in separability is observed after normalized differential ratio processing.

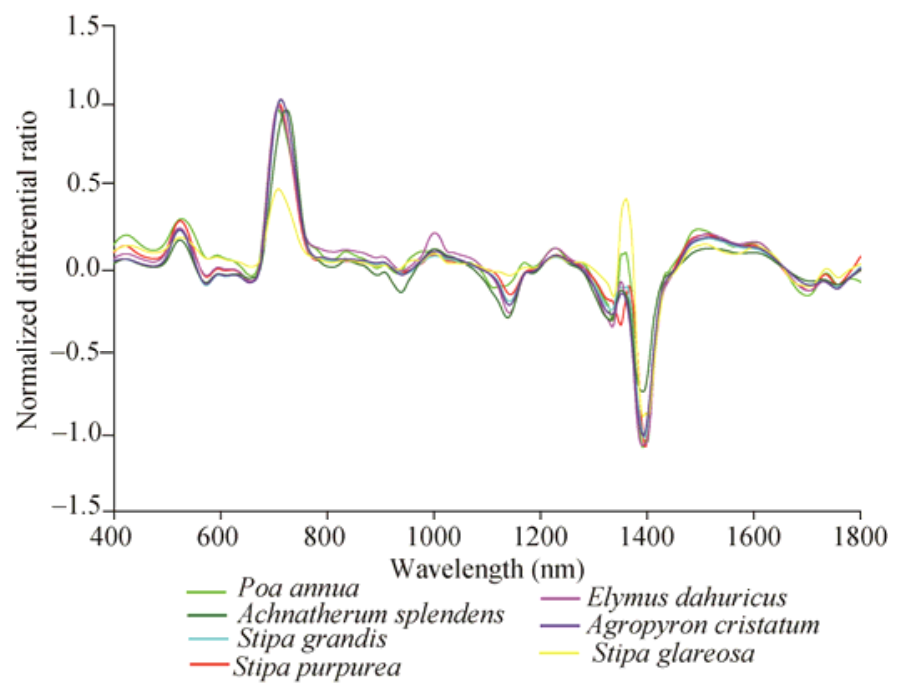

Fig. 5 Normalized differential ratios of the typical rolled-leaf desert plant species in the Hexi Corridor

\subsection{Rolled-leaf desert plant identification and accuracy verification}

Based on this study of spectral characteristics, we proposed and verified two schemes for the identification of typical rolled-leaf desert plants in the Hexi Corridor using hyper-spectral data. The flow diagrams for both schemes are shown in Figure 6 and are described below.

Scheme A

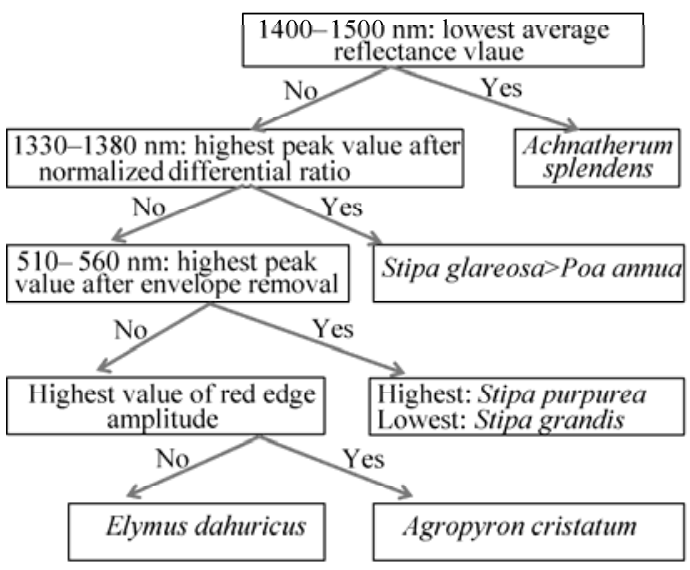

Scheme B

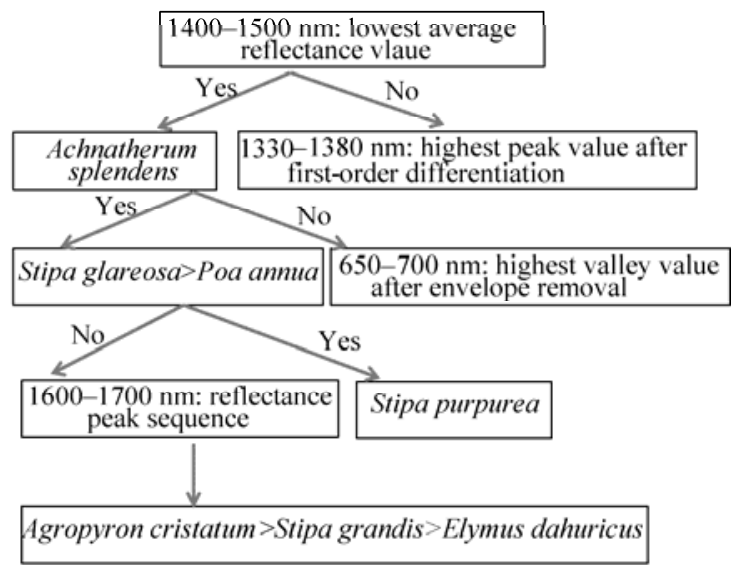

Fig. 6 Flow diagrams of the identification of the typical rolled-leaf desert plant species using the hyper-spectral data 
Scheme A: (1) in the 1400-1500 nm regions, Achnatherum splendens has the lowest average spectral reflectance value; (2) after normalized differential ratio processing of the spectral curves, Stipa glareosa has the highest value in the 1330-1380 nm regions, followed by Poa annua; (3) after removing Achnatherum splendens, Stipa glareosa, and Poa annua, and using the normalized envelope method to process the spectral curves, Stipa purpurea has the highest value in the 510-560 nm regions, and Stipa grandis has the lowest value; and (4) after removing the above five plant species, the red edge amplitude of Agropyron cristatum exceeds that of Elymus dahuricus. The plant identification sequence in Scheme A is as follows: Achnatherum splendens, Stipa glareosa, Poa annua, Stipa purpurea, Stipa grandis, Agropyron cristatum, and Elymus dahuricus.

Scheme B: (1) in the 1400-1500 nm regions, Achnatherum splendens has the lowest average spectral reflectance value; (2) after first-order differential mathematical transformation of the spectral curves, Stipa glareosa has the highest peak value in the 1330-1380 nm regions, followed by Poa annua; (3) after removing Achnatherum splendens, Stipa glareosa, and Poa annua, and using the normalized envelope method to process the spectral curves, Stipa purpurea has the highest valley value in the 650-700 nm regions; and (4) after removing these four vegetation species, Agropyron cristatum has the highest reflectance value in the 1600-1700 nm regions, followed by Stipa grandis, while Elymus dahuricus has the lowest value. The vegetation identification sequence in Scheme B is as follows: Achnatherum splendens, Stipa glareosa, Poa annua, Stipa purpurea, Agropyron cristatum, Stipa grandis, and Elymus dahuricus.

We randomly selected three groups of hyper-spectral reflectance data (Figs. 7a-c) from each of the twenty rolled-leaf desert plant identification test samples. We obtained the average values of the three sets of data (Fig. 7d) and analyzed the spectral reflectance characteristics. The three-edge parameters were then extracted (Table 3), and the normalized differential ratio was applied, followed by envelope normalization processing of the reflectance data.
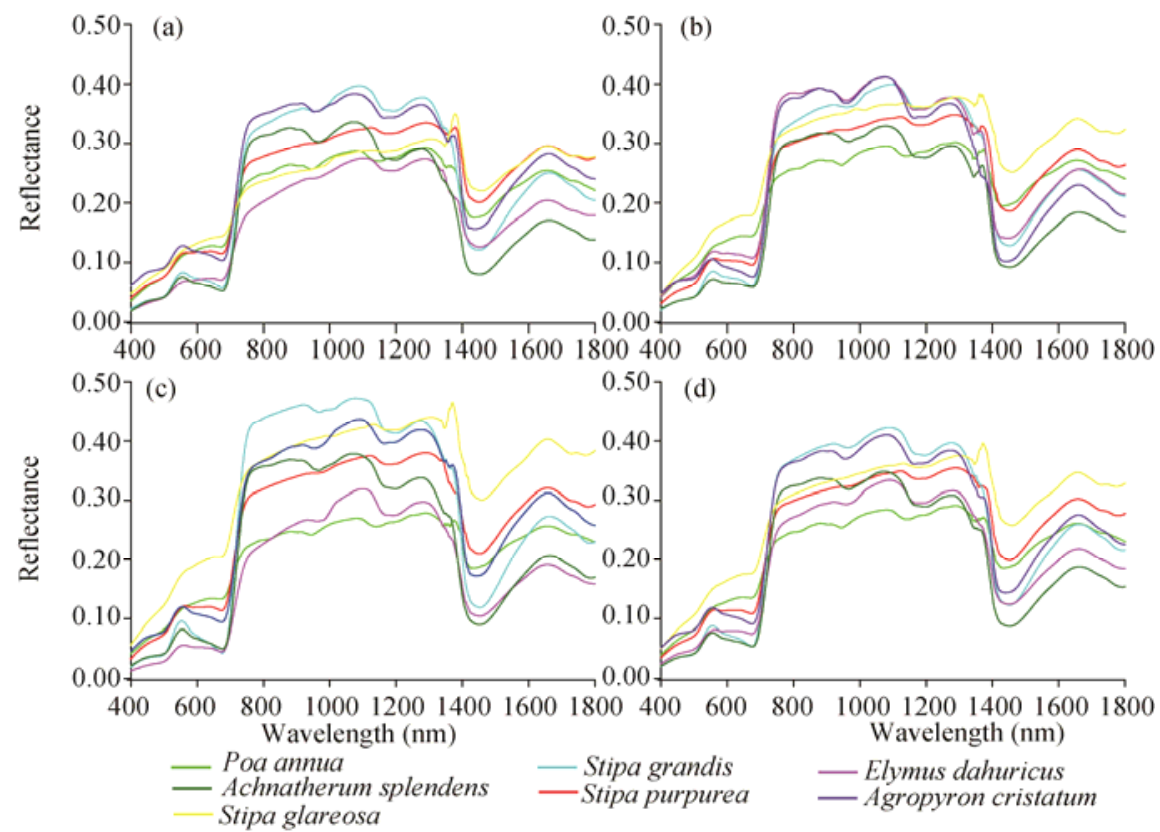

Fig. 7 Selected three groups of hyper-spectral reflectance curves of the typical rolled-leaf desert plant species $(\mathrm{a}-\mathrm{c})$ and their average values $(\mathrm{d})$

Figure 7 shows that the spectral reflectance curve data for the rolled-leaf desert vegetation groups a, b, and c can be distinguished relatively well in the 1400-1800 $\mathrm{nm}$ regions and especially in the $1400-1500$ and $1600-1700 \mathrm{~nm}$ regions. During processing the spectral reflectance curves, the peak values in the 1330-1380 $\mathrm{nm}$ regions and the red edge amplitude, the valley values in the 510-560 $\mathrm{nm}$ regions and the peak values in the $650-700 \mathrm{~nm}$ regions, we find that there are also differences between the spectral curves of different rolled-leaf desert plant types (Table 3 ). 
WEI Huaidong et al.: Hyper-spectral characteristics of rolled-leaf desert vegetation in the Hexi Corridor...

Table 3 Red-edge parameter statistics of the typical rolled-leaf desert plant types $\left(\times 10^{-3}\right)$

\begin{tabular}{|c|c|c|c|c|c|c|c|}
\hline Test group & $\begin{array}{c}\text { Poa } \\
\text { annua }\end{array}$ & $\begin{array}{c}\text { Stipa } \\
\text { purpurea }\end{array}$ & $\begin{array}{c}\text { Stipa } \\
\text { grandis }\end{array}$ & $\begin{array}{c}\text { Elymus } \\
\text { dahuricus }\end{array}$ & $\begin{array}{c}\text { Achnatherum } \\
\text { splendens }\end{array}$ & $\begin{array}{c}\text { Stipa } \\
\text { glareosa }\end{array}$ & $\begin{array}{c}\text { Agropyron } \\
\text { cristatum }\end{array}$ \\
\hline $\mathrm{a}$ & 2.2 & 3.0 & 4.7 & 2.2 & 4.8 & 1.6 & 4.3 \\
\hline $\mathrm{b}$ & 2.1 & 3.7 & 4.7 & 5.4 & 4.6 & 2.6 & 5.7 \\
\hline $\mathrm{c}$ & 1.9 & 3.7 & 7.3 & 2.9 & 5.7 & 3.0 & 4.8 \\
\hline $\mathrm{d}$ & 2.1 & 3.5 & 5.6 & 3.5 & 5.0 & 2.4 & 4.9 \\
\hline
\end{tabular}

Note: Group d comprises the average values of the three preceding groups a-c.

To ensure that the selected bands, 1400-1500, 1330-1380, 510-560, and 650-700 nm, are the optimal regions for distinguishing different species of rolled-leaf desert plants, we used independent-samples test methods, Kruskal-Waillis test and Median test to check whether significant differences existed among these spectrum regions (Table 4). The result shows that these spectrum regions are useful for different rolled-leaf plant species in the Hexi Corridor. All four regions pass both the tests. This indicates that $1400-1500$ and $1600-1700 \mathrm{~nm}$ regions are the optimal bands for all types of rolled-leaf desert plant species, while the 1330-1380, 510-560, and 650-700 $\mathrm{nm}$ regions could facilitate the differentiation of the seven rolled-leaf desert plant species considered in this study.

Table 4 Significant differences test of the rolled-leaf desert plants in selected spectrum regions

\begin{tabular}{cccccc}
\hline & \multicolumn{5}{c}{$P$ value } \\
\cline { 2 - 6 } & $1400-1500 \mathrm{~nm}$ & $1600-1700 \mathrm{~nm}$ & $510-560 \mathrm{~nm}$ & $650-700 \mathrm{~nm}$ & $1330-1380 \mathrm{~nm}$ \\
\hline Kruskal-Waillis test & 0.000 & 0.000 & 0.000 & 0.000 & 0.000 \\
Median test & 0.000 & 0.001 & 0.000 & 0.000 & 0.001 \\
\hline
\end{tabular}

Using schemes $\mathrm{A}$ and $\mathrm{B}$, we carried out classification and identification of the randomly selected rolled-leaf desert vegetation groups a, b, and c, as well as their average values d. Based on the accuracy rate statistics (Table 5), we found that both schemes have $100 \%$ identification accuracy for Achnatherum splendens, Stipa glareosa, and Poa annua. Overall, Scheme A has $100 \%$ identification accuracy, and Scheme B has an accuracy of greater than $95 \%$. This shows that the selection of the spectral characteristic bands for identification of the seven typical rolled-leaf desert plant types in the Hexi Corridor in this study is effective, and that both schemes can be used successfully.

\section{Discussion}

\subsection{Importance of desert vegetation water content in spectral identification}

The four main absorption regions of liquid water are located at the 970, 1200, 1450, and $1930 \mathrm{~nm}$ band positions, respectively, which is similar to the reflectance spectrum of vegetation leaves and canopy (Gates et al., 1965). Some studies have suggested that the 1405-1646 nm bands are sensitive to moisture in desert vegetation and can reflect its water content (Zhao, 2011). However, other studies have suggested that the 1374-1534 nm bands show the best correlation with the water content of desert plants and are the characteristic bands for expressing water content in vegetation (Zhao et al., 2010). In this study, we find that the spectral characteristics of rolled-leaf desert plants are well-distinguishable in the 1400-1800 $\mathrm{nm}$ regions, within which the absorption valleys around the 1400-1500 nm intervals and the reflectance peaks around the 1600-1700 nm intervals are more distinguishable on the spectral curves of various plant types.

After processing the spectral data using the normalized differential ratio and the first-order differential algorithm, the spectral characteristics of some types of rolled-leaf desert plant become more evident in the 1330-1380 $\mathrm{nm}$ regions. The above characteristic bands, which are located in 
Table 5 Statistically analyzed accuracy of the rolled-leaf desert plant type identification

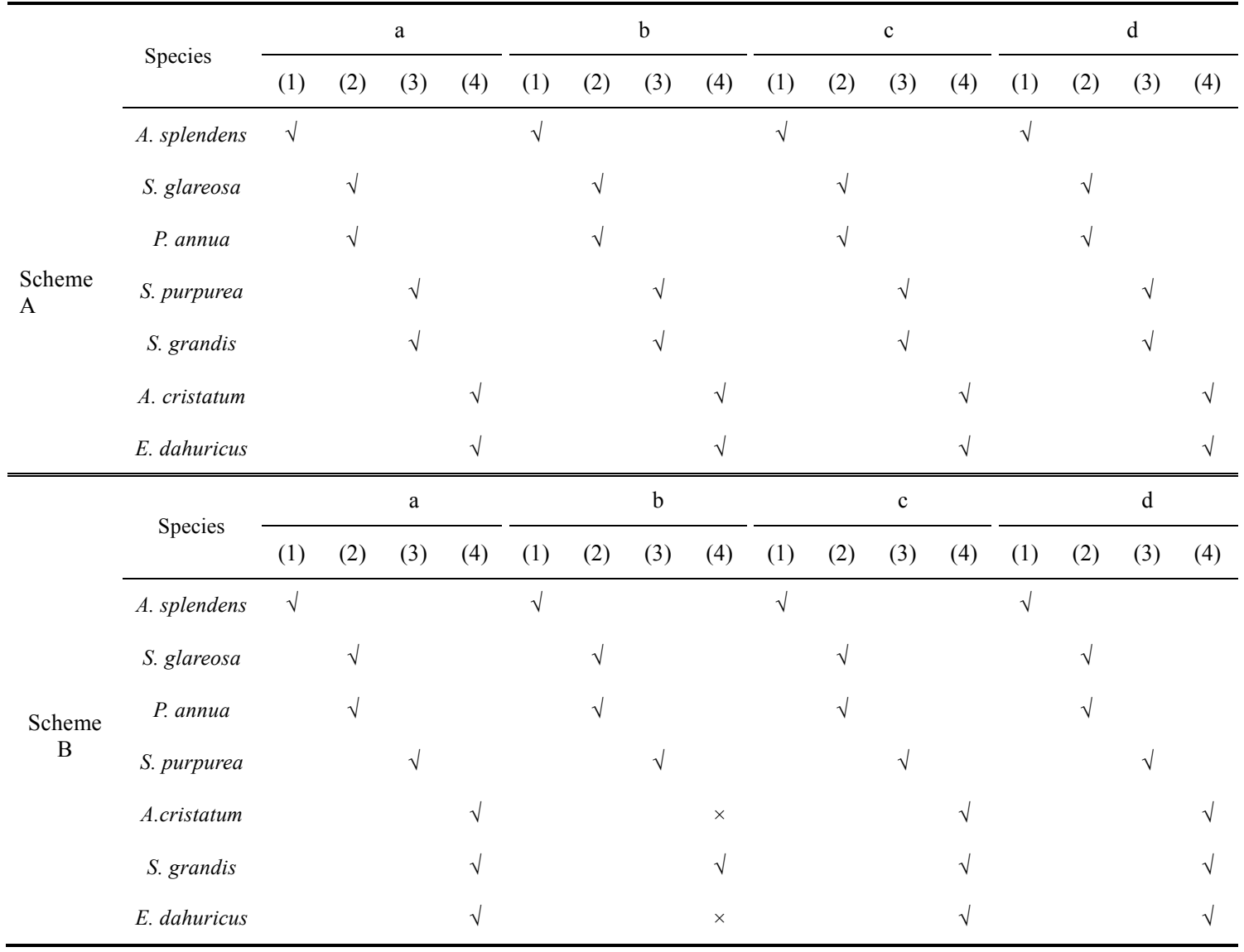

Note: a-d indicate the test groups; (1)-(4) indicate identification attempt; $\sqrt{ }$ indicates correct identification; and $\times$ indicates incorrect identification.

the reflectance region controlled by the leaf water content $(1250-2300 \mathrm{~nm})$, are used in the identification schemes for the rolled-leaf desert plants in this study with good results. This shows that water content is a very important parameter for spectral identification of rolled-leaf desert plants. Prevention of water loss and reducing transpiration are the main strategies used by desert vegetation to adapt to the arid environment. Therefore, it is necessary to further explore the correlation between the reflectance of different bands and the water content of desert vegetation. Combining the vegetation water content and other indicators to study the spectral and discriminative characteristics of desert plant species, and using hyper-spectral data to find a more suitable vegetation index that can represent the vegetation water content (Mirzaie et al., 2014) can provide a reference for monitoring and species identification in deserts.

\subsection{Implications for accurate hyper-spectral identification of desert vegetation}

One of the advantages of hyper-spectral data is the fine extraction and identification of target characteristics, and the selection of these characteristics is a key issue in its application (An, 2014). Through processing and analysis of hyper-spectral field data, we gain a better understanding of the spectral characteristics and variation patterns of vegetation communities, and improve the accuracy of vegetation identification, with good results obtained (Zhang, 2006; Gai et al., 2011). Although good results are obtained, a larger sample size may increase the difficulty of identification. The next step is to combine the desert vegetation water content, chlorophyll content, canopy transpiration, tissue structure, and other indicators to further explore the characteristic bands of various types of desert plant. Due to the harsh natural selection process, desert plants possess many unique properties, such as having many branches and few leaves, or having mostly needle-like or thorn-like leaves. The hyper-spectral characteristics of desert plants 
are complex, and widely different spatial characteristics can appear even for the same type of plant in different seasons. Therefore, various phenological spectral observations can be combined with diverse vegetation indices and other indicators, to carry out a study on more specific classification of vegetation. In addition, due to low desert vegetation coverage and the significant effect that soil background has on the spectral data, finding ways to improve the detection or elimination of background information, and combining the field mixed spectral data and pure spectral information or the canopy and leaf spectral information for vegetation identification studies, are currently some of the most important and difficult areas of research related to extraction and recognition of the spectral characteristics of desert vegetation.

\section{Conclusions}

During the vigorous vegetation growth period in July and August, the locations of the red valleys $(650-690 \mathrm{~nm})$, green peaks $(510-560 \mathrm{~nm})$, and three-edge parameters, namely, the red edge $(680-760 \mathrm{~nm})$, the blue edge $(490-530 \mathrm{~nm})$, and the yellow edge $(560-640 \mathrm{~nm})$, of seven typical rolled-leaf desert plant types in the Hexi Corridor, are essentially consistent with those of the majority of terrestrial vegetation types. There are less than $20 \%$ of green peaks in the visible light spectrum. Based on the analysis of hyper-spectral reflectance data, we consider that the main absorption regions of liquid water, i.e., 1400-1500 and 1600-1700 nm, are the optimal bands for distinguishing various types of rolled-leaf desert plant. In contrast, it is difficult to select the characteristic bands for differentiation of the various plant types in the leaf reflectance region, which is controlled by the cellular structure, namely, the 700-1250 nm regions. After processing the spectral reflectance curves using various mathematical transformation methods, including first-order differential, envelope removal, and the normalized differential ratio, some parameters that are useful for improving identification ability using the hyper-spectral characteristics of rolled-leaf desert plants have been identified. These are the 1330-1380, 510-560 and 650-700 nm regions, and the red edge amplitude. Based on the analysis of hyper-spectral reflectance characteristics, we proposed two schemes for identification of various types of rolled-leaf desert plant in the Hexi Corridor. The identification accuracy rates of these two schemes exceeded 95\%, even reached $100 \%$ with regards to Achnatherum splendens, Stipa glareosa, and Poa annua species. We conclude that the selection of characteristic bands for identification of typical rolled-leaf desert plant types in the Hexi Corridor in this study is effective, and that the identification schemes can be used successfully.

\section{Acknowledgements}

This study was supported by the National Natural Science Foundation of China (31760241, 41671528), the Gansu Provincial Natural Science Foundation (17JR5RA061), the Gansu Province Basic Research Innovation Group Project (1506RJIA155), and the Opening Foundation of the State Key Laboratory Breeding Base of Desertification and Aeolian Sand Disaster Combating, Gansu Desert Control Research Institute (GSDC201503). Thanks to the State Key Laboratory of Desertification and Sandstorm Prevention and Control of Gansu Province, and the Minqin National Field Station for Desert Steppe Ecosystem Studies in Gansu Province for providing support in the field investigation.

\section{References}

Adam E, Mutanga O. 2009. Spectral discrimination of papyrus vegetation (Cyperus papyrus L.) in swamp wetlands using field spectrometry. ISPRS Journal of Photogrammetry and Remote Sensing, 64(6): 612-620.

An R, Jiang D P, Li X X, et al. 2014. Using hyperspectral data to determine spectral characteristics of grassland vegetation in central and eastern parts of Three-river Source. Remote Sensing Technology and Application, 29(2): 202-211. (in Chinese)

Cao W, Shao Q Q, Yu X Y, et al. 2013. Analysis of spectral characteristics of Inner Mongolia's temperature steppe in different use patterns. Acta Agrestia Sinica, 21(2): 243-252. (in Chinese)

Fang M H, Ju W M, Zhan W F, et al. 2017. A new spectral similarity water index for the estimation of leaf water content from hyperspectral data of leaves. Remote Sensing of Environment, 196: 13-27.

Gai Y Y, Fan W J, Xu X R, et al. 2011. Flower species identification and coverage estimation based on hyperspectral remote 
sensing data in Hulunbeir grasslasnd. Spectroscopy and Spectral Analysis, 31(10): 2778-2783. (in Chinese)

Gates D M, Keegan H J, Schleter J C, et al. 1965. Spectral properties of plants. Applied Optics, 4(4): 11-20.

He L, An S Z, Jin G L, et al. 2014. Analysis on high spectral characteristics of degraded Seriphidium transiliense desert grassland. Acta Agrestia Sinica, 22(2): 271-276. (in Chinese)

Jin G L, He L, An S Z, et al. 2014. Spectral features of eight desert range plants on degradation Seriphidium transiliense desert grassland. Pratacultural Science, 31(10): 1848-1858. (in Chinese)

Jin J, Wang Q. 2016. Hyperspectral indices based on first derivative spectra closely trace canopy transpiration in a desert plant. Ecological Informatics, 35: 1-8.

Li X S. 2008. Quantitative retrieval of sparse vegetation cover in arid regions using hyperspectral data. PhD Dissertation. Beijing: Chinese Academy of Forestry. (in Chinese)

Liu N F, Budkewitsch P, Treitz P. 2017. Examining spectral reflectance features related to Arctic percent vegetation cover: Implications for hyperspectral remote sensing of Arctic tundra. Remote Sensing of Environment, 192: 58-72.

Mansour K, Mutanga O, Everson T, et al. 2012. Discriminating indicator grass species for rangeland degradation assessment using hyperspectral data resampled to AISA Eagle resolution. ISPRS Journal of Photogrammetry and Remote Sensing, 70: $56-65$.

Mansour K, Mutanga O, Everson T. 2013. Spectral discrimination of increaser species as an indicator of rangeland degradation using field spectrometry. Journal of Spatial Science, 58(1): 101-117.

Meyer H, Lehnert L W, Wang Y, et al. 2017. From local spectral measurements to maps of vegetation cover and biomass on the Qinghai-Tibet-Plateau: Do we need hyperspectral information? International Journal of Applied Earth Observation \& Geoinformation, 55: 21-31.

Mirzaie M, Darvishzadeh R, Shakiba A, et al. 2014. Comparative analysis of different uni- and multi-variate methods for estimation of vegetation water content using hyper-spectral measurements. International Journal of Applied Earth Observation and Geoinformation, 26: 1-11.

Qian Y R, Yu J, Jia Z H, et al. 2013. Extraction and analysis of hyper spectral data from typical desert grassland in Xinjiang. Acta Prataculturae Sinica, 22(1): 157-166. (in Chinese)

Schmidt K S, Skidmore A K. 2003. Spectral discrimination of vegetation types in a coastal wetland. Remote Sensing of Environment, 85(1): 92-108.

Wei X H, Jin G L, Fan Y M, et al. 2016. Species analysis and identification of spectral characteristics on Seriphidium transiliense desert grassland. Pratacultural Sciende, 33(10): 1924-1932. (in Chinese)

Xia X W, Jin G L, An S Z, et al. 2015. Spectral characteristics of typical plants in Seriphidium transiliense desert grassland under enclosure. Pratacultural Science, 32(6): 870-876. (in Chinese)

Yang K, Shen W S, Bo L. 2014. Research on spectral reflectance characteristics for Naqu typical grassland. Remote Sensing Technology and Application, 29(1): 40-45. (in Chinese)

Yang X M, Liu S Z, Yang T B, et al. 2016. Spatial-temporal dynamics of desert vegetation and its responses to climatic variations over the last three decades: a case study of Hexi region in Northwest China. Journal of Arid Land, 8(4): 556-568.

Zarco-Tejada P J, Hornero A, Hernández-Clemente R, et al. 2018. Understanding the temporal dimension of the red-edge spectral region for forest decline detection using high-resolution hyperspectral and Sentinel-2a imagery. ISPRS Journal of Photogrammetry and Remote Sensing, 137: 134-148.

Zhao J, Chen X, Guli J, et al. 2009. Spectral discrimination of desert vegetation in the Tarim River basin. Journal of Desert Research, 29(2): 270-278. (in Chinese)

Zhao Z, Li X, Yin Y B, et al. 2010. Analysis of spectral features based on water content of desert vegetation. Spectroscopy and Spectral Analysis, 30(9): 2500-2503. (in Chinese)

Zhao Z. 2011. Analysis of spectral features based on water content of Xinjiang desert plants. MSc Thesis. Xinjiang: Xinjiang Agricultural University. (in Chinese)

Zhang C M, Zhang J M. 2012. Research on the spectral characteristics of grassland in arid regions based on hyperspectral image. Spectroscopy and Spectral Analysis, 32(2): 445-448.

Zhang F, Tashpolat T, Ding J L, et al. 2012. Spectral reflectance characteristics of typical halophytes in the oasis salinization desert zone of middle reaches of Tarim River, China. Chinese Journal of Plant Ecology, 36(7): 607-617. (in Chinese)

Zhang K, Guo N, Wang R Y, et al. 2006. Research on spectral reflectance characteristics for desert meadow of Northwest China. Advances in Earth Science, 21(10): 1063-1069. (in Chinese)

Zhou L P, Wei H D, Ding F, et al. 2013. Analysis on spectral reflectance characteristics of desert plants in Minqin basin of Shiyang River. Journal of Arid Land Resources and Environment, 27(3): 121-125. (in Chinese) 